\title{
Intelligent Traffic Incidents Detection Method in Freeway Corridors
}

\author{
Binbin Zhou, Hexin Lv, Tiaojuan Ren, Yourong Chen and Ning Qiu \\ College of Information Science and Technology \\ Zhejiang Shuren University \\ Hangzhou, China \\ e-mail: bzhou@zjsru.edu.cn, hexin1024@sohu.com, kuanren988@sina.com,jack_chenyr@163.com,7674060@qq.com
}

\begin{abstract}
Traffic incidents detection has become an important issue in the past decades, which would result in a large amount financial cost and considerable reduction in traffic efficiency. Most previous studies mainly pay their attention on automatic incident detection in freeway corridors based on the traffic data collected by inductive loops or video monitors. In this paper, we have presented a support vector machines (SVM)-based method to confirm whether a traffic incident occurs or incident-free in freeway corridors with the utilization of the real-time traffic data collected and transmitted by wireless sensors. We also have extracted the most crucial traffic variables as features and utilized them into the SVM model for data process. Several experiments have been conducted to evaluate our method's performance compared with a representative relevant work, in terms of detection rate, mean time-to-detect and false alarm rate. The experimental results depict that our method could obtain better performance in most case.
\end{abstract}

Keywords-traffic incident detection; freeway corridors; intelligent detection; wireless sensors; support vector machines (SVM)

\section{INTRODUCTION}

Traffic congestion has become a wide spreading and growing problem today, because of the existing transportation infrastructure are unable to provide sufficient support. Traffic incidents plays an important part to cause traffic jam, which refer to events happened abnormally in regular and will cause lower traffic efficiency and longer vehicles flow waiting, such as traffic accidents [1]. Therefore, the traffic incident detection has emerged in the past years, and attracted abundant research scholars' attention. Effective incident detection methods can be useful to ease traffic congestion and reduce drivers' travelling durations. The traditional traffic incident detection methods mainly focus on using inductive loop traffic data and video traffic data, with lots of shortcoming, such as limited detection range and high costs of implementation and maintenance. Therefore, we choose to use the wireless sensor nodes, to detect the real-time traffic data.

There has been a large amount studies adopting some techniques for traffic incident detection in recent years [2][16]. Previous traffic incident detection methods are mainly applied into two fields, freeway corridors and urban roads with different traffic characteristics. From the aspect of techniques, existing studies can be categorized into four groups, machine learning (ML)-inspired algorithms, time series analysis (TSA), other comparative methods and hybrid methods. ML-based methods focus on traffic patterns and estimate current detected traffic variables whether it's incident-free [3]-[8]. TSA methods underline dynamic and abnormal changes of traffic [9][12]. There are also some comparative methods [13] [14] and hybrid methods [15] [16].

In this paper, we choose wireless sensors to detect traffic data in a real-time manner, and then adopt support vector machines to deal with the fused traffic data, to obtain the final decision result, whether an incident occurs. SVM are effective tools in a broad area of classification problems and robust to irrelevant features [17]. We extract the most important features (e.g. traffic volume and average traffic flow speed) related to incidents occurrences, and then train the SVM model through various features combinations. At last, we conduct experiments to evaluate our method's performance based on a publicly available dataset. And the experimental results depict that our method can obtain better performance in terms of detection rate, mean time to detect and false alarm rate.

The remainder of this paper is organized as follows. Section II describes the problem formulation. Section III explains our SVM-based mechanism in details. Section IV elaborates the design of experiments and results. Finally, Section V concludes the paper and outlines future research directions.

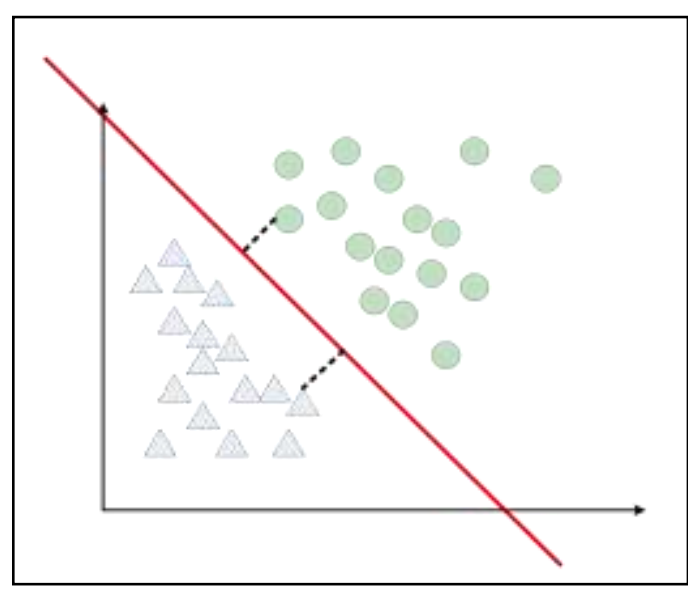

Figure 1. A binary classification problem 


\section{PROBLEM FORMULATION}

The problem of traffic incident detection is how to confirm the infrequent traffic situation from large amount traffic situations, to decide whether a traffic incident occurs or incident-free. It is similar to the binary classification problem as shown in Fig.1. Our objective is to find the red line to separate these green circles and blue tri-angles into different sides. In this way, when some traffic variables are detected real-timely and inducing a traffic situation deviation with regular traffic patterns, we can utilize the red line to confirm which side these traffic variables should take place in.

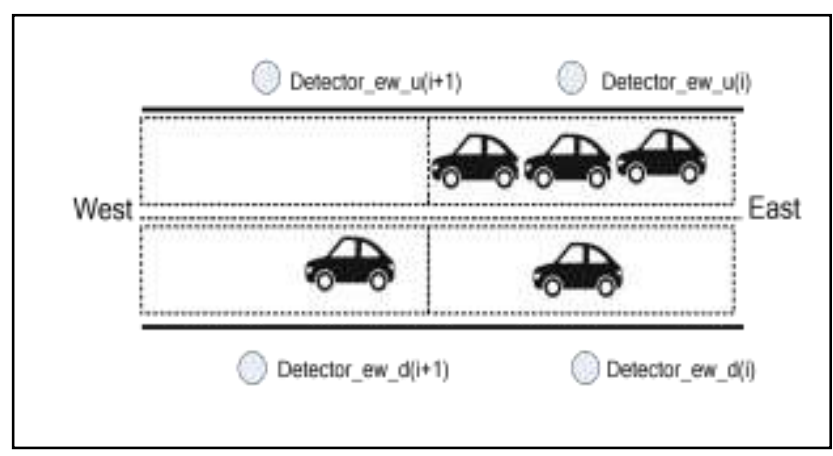

Figure 2. A Traffic Scenario Model

To model this problem, we would consider a detector-equipped freeway road scenario (see Fig .2) which divided into several segments due to detector's detection range. We assume that when each vehicle comes into a road segment, the corresponding detector can sense its existence successfully.

TABLE I. NOTATION EXPLANATION

\begin{tabular}{|l|c|}
\hline \multicolumn{1}{|c|}{ Notation } & Definition \\
\hline$i$ & Detector node on the corridor \\
\hline $\mathbf{x}(\mathbf{i})$ & $\begin{array}{c}\text { Road segment vector covered by } \\
\text { node } i\end{array}$ \\
\hline$y(i)$ & $\begin{array}{c}\text { Decision result of road segment } \\
\text { labeled by } i\end{array}$ \\
\hline Detector_ew_u(i) & $\begin{array}{c}\text { Nodes installed in the upper side of } \\
\text { road segment from east to west }\end{array}$ \\
\hline Detector_ew_d(i) & $\begin{array}{c}\text { Nodes installed in the downside of } \\
\text { road segment from east to west }\end{array}$ \\
\hline
\end{tabular}

We define some notations for this problem as shown in Table I. We represent traffic variables in different segments as vectors $\mathbf{x}(i), i=1,2,3, \ldots, N$. Each $\mathbf{x}(i)$ has its own final result, $y(i), y(i) \in\{1,-1\}$. Our objective is to find a function $\mathrm{F}$ in the following expression.

$$
\mathrm{F}: \mathbf{x} \rightarrow y
$$

\section{INTELLIGENT INCIDENT DETECTION METHOD}

In this section, we propose our intelligent traffic incident detection method based on the above model. The work flow of our method is presented in Fig .3. There are five steps, traffic data detection, traffic data preprocess, traffic data fusion, traffic data utilization in SVM model, and situation determination.
When detecting traffic data, sensors equipped roadside are usually the popular choices due to its convenient deployment and maintenance, such as wireless sensors. After the real-time traffic data collected, they need to be preprocessed in order to adapt to SVM model. In this model, when an incident happens in a segment, traffic volume of this segment and following segments would grow rapidly, with tangible reduction in the segments ahead. Similar change trends would occur on segment occupancy. In terms of average traffic flow speed, the speed of this segment and following segments would decrease obviously, with distinct improvement in the segments ahead. Hence, we decide to treat both traffic volume difference and speed difference between current segment and segment ahead as input variables for the SVM model, which means the data preprocess part should finish this job when receive all the traffic data collected. The detail mechanism of the SVM model would be presented in following. Based on the output of the SVM model, we can confirm whether an incident happens.

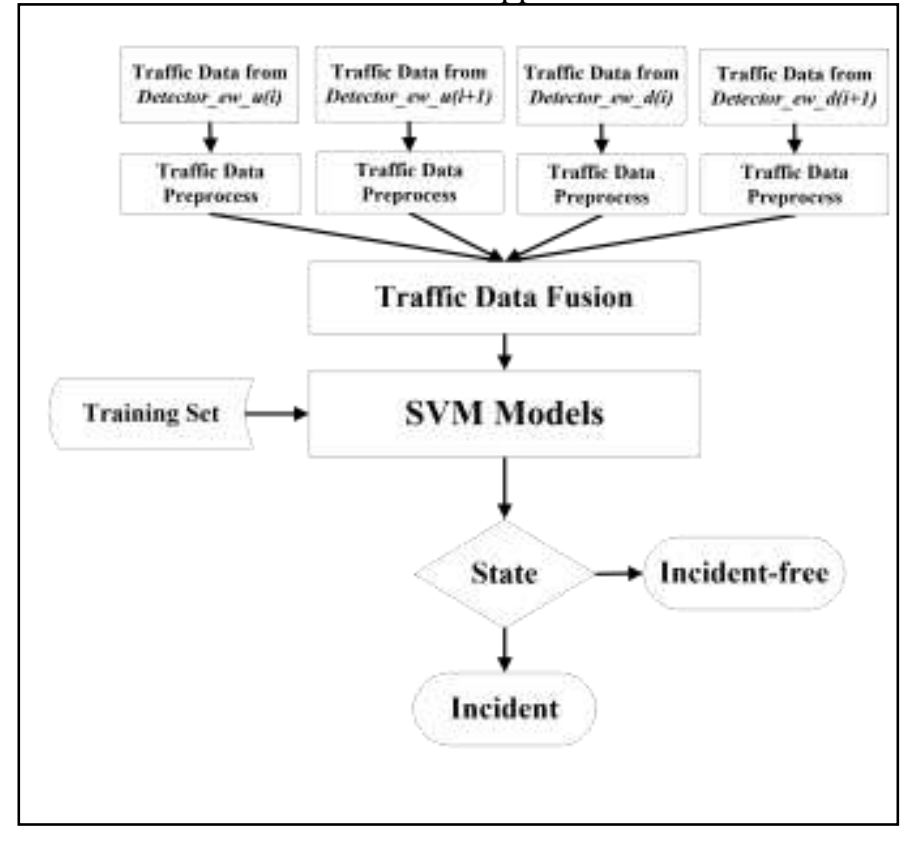

Figure 3. Work Flow of Intelligent Traffic Incident Detection

Based on the analysis mentioned above, vector $\mathbf{x}(i)$ has two elements, traffic volume difference between segment $i$ and segment ahead $i+1$, defined as $t_{V d}(i, i+1)$, and speed difference between segment $i$ and segment ahead $i+1$, defined as $s d(i, i+1)$.

$$
\begin{gathered}
x(i)=(\operatorname{tvd}(i, i+1), s d(i, i+1))^{T} \\
i=1,2,3, \ldots, N
\end{gathered}
$$

The objective is finding a maximum-margin hyper plane $\boldsymbol{\omega} \cdot \mathbf{x}+b=0$, which divides the variables having $y(i)=1$ from those having $y(i)=-1$. With a non-negative slack variable $\xi_{i}$ to avoid mislabeled instances, the optimization problem can be presented as follows. 


$$
\min _{\omega, b, \xi} \frac{1}{2}\|\omega\|^{2}+C \sum_{i=1}^{N} \xi_{i}
$$

$$
\begin{array}{r}
\text { s.t. } \quad y_{i}\left(\boldsymbol{\omega} \cdot \mathbf{x}_{\mathrm{i}}+b\right) \geq \\
\xi_{i} \geq 0, i=\xi_{i}, i=1,2,3, \ldots, N \\
\end{array}
$$

The SVM soft margin maximization problem can be transferred to its corresponding dual problem. And its purpose is to find the optimal $\alpha^{*}=\left(\alpha_{1}^{*}, \alpha_{2}{ }^{*}, \mathrm{~K}, \alpha_{N}{ }^{*}\right)^{T}$, which can satisfy the following formula.

$$
\begin{aligned}
\min _{\alpha} & \frac{1}{2} \sum_{i=1}^{N} \sum_{j=1}^{N} \alpha_{i} \alpha_{j} y_{i} y_{j}\left\langle x_{i}, x_{j}\right\rangle-\sum_{i=1}^{N} \alpha_{i} \\
\text { s.t. } \quad & \sum_{i=1}^{N} \alpha_{i} y_{i}=0 \\
& 0 \leq \alpha_{i} \leq C, i=1,2,3, \ldots, N
\end{aligned}
$$

After the optimal solution obtained, and then

$$
\begin{gathered}
\boldsymbol{\omega}^{*}=\sum_{i=1}^{N} \alpha_{i}^{*} y_{i} \mathbf{x}_{\mathrm{i}} \\
b^{*}=y_{i}-\sum_{i=1}^{N} y_{i} \alpha_{i}^{*}\left\langle\mathbf{x}_{\mathrm{i}}, \mathbf{x}_{\mathbf{j}}\right\rangle
\end{gathered}
$$

Thus,

$$
F(\mathbf{x})=\operatorname{sign}\left(\mathbf{\omega}^{*} \cdot \mathbf{x}+b^{*}\right)
$$

\section{EXPERIMENTS AND ANALYSIS}

\section{A. Experiment Data Set and Evaluation Metrics}

The traffic dataset used for experiments is derived from the publicly available I-880 database from the Freeway Patrol Service Project in California, USA [18][19]. The data are collected during two periods: from February 16, 1993 to March 19, 1993 and from September 27, 1993, to October 29, 1993. This dataset includes the traffic data we demand for, such as traffic volume (in vehicles per hour) and speed (in miles per hour). And they also include abundant incidents events, almost 45 laneblocking incidents [16].

The most common and widely acknowledge evaluation metrics for traffic incident detection are detection rate (DR), false alarm rate (FAR), and mean time to detect (MTTD). DR is defined as the ratio of the number of correctly detected incidents to the number of all incidents, presented in formula 8. FAR is defined as the ratio of the number of false decisions to the number of all incident-free cases, and presented in formula 9. MTTD is defined as the average of each time periods cost from the moment an incident occurs and the moment the incident detected, and presented in formula $10, N$ is defined as the total incident number.

$$
\begin{array}{r}
D \mathrm{R}=\frac{\# \text { of correctly detected incidents }}{\# \text { of all incidents }} \times 100 \% \\
F \mathrm{AR}=\frac{\# \text { of false decisions }}{\# \text { of all incident-free cases }} \times 100 \% \\
\text { MTTD }=\sum_{i=1}^{N} \frac{T_{\text {detection }}(i)-T_{\text {incident }}(i)}{N}
\end{array}
$$

\section{B. Experimental Design and Analysis}

In traffic incidents detection problems, we would prefer higher DR, lower FAR, and shorter MTTD, which leads to a multi-purposes problem. The three goals are difficult to achieve optimal solution simultaneously. A higher DR may cause higher FAR and longer MTTD. Hence, we evaluate the performance separately, DR versus FAR and MTTD versus FAR respectively. Since our method is based on SVM and select different features in the training stage, we adopt a representative related work [5] as the comparative method.

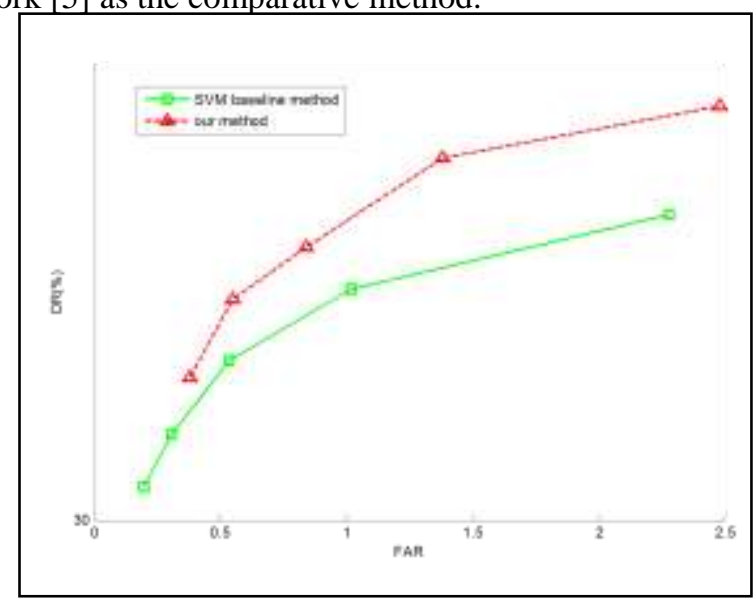

Figure 4. DR Comparison between the two methods

Fig .4 presents the detection rate comparison between a SVM baseline method [5] and our proposed method. From the figure, we can observe that our method can outperform the other method in most cases. When the FAR lower than $0.5 \%$, the SVM baseline method presents the best performance. With the incidents number increases, both methods have witnessed higher FAR, companied with higher DR. At that time, our method obtains better performance and expands the difference. 


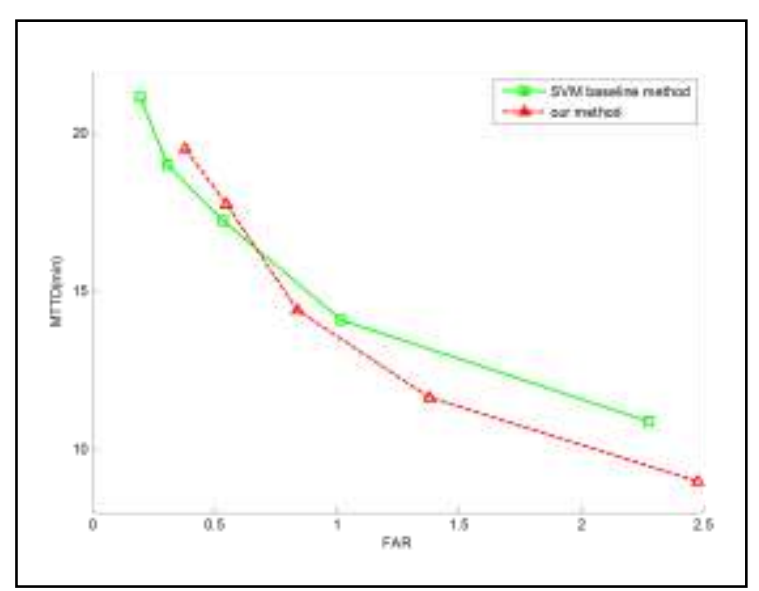

Figure 5. MTTD Comparison between the two methods

Fig .5 presents the mean time-to-detect comparison between these two methods. From the figure, we can notice that with both methods has different performance when with different FAR. When the FAR is lower than $0.7 \%$, the method from [5] achieves better performance with much lower MTTD. When the FAR is higher $0.7 \%$, our method can outperform the other method.

\section{V.CONCLUSION}

Nowadays, traffic congestion problems have increasingly attracted people's attention due to the huge financial cost and air pollutions. Traffic incidents have been pointed out that plays an important part in traffic congestion. In this paper, we have presented an intelligent traffic incident detection method based on SVM with appropriate features, with traffic data detected by wireless sensor in a real-time manner. After several experiments conducted based on a real-world dataset, we confirm that our features selected can be beneficial for incidents detection, with higher detection rate and low mean time-todetect with a certain level FAR, compared with another representative related work. In the future, we will optimize our work to further improve the detection rate, and we would pay efforts to optimize current method in order to apply into urban areas.

\section{ACKNOWLEDGMENT}

This work was supported in part by Public Welfare Technology Applied Research Program of Zhejiang Province (2014C33108) and Zhejiang Provincial Natural Science Foundation of China (LY13F010013).

\section{REFERENCES}

[1] F. Y. Wang, "Parallel control and management for intelligent transportation systems: Concepts, architectures, and applications," IEEE Trans. Intell. Transp. Syst., vol. 11, no. 3, pp. 630-638, Sep. 2010.
[2] J. Luk, C. Han, and D. Chin, Freeway Incident DetectionTechnologies and Techniques. Sydney, Australia: Austroads, 2010, p. 72.

[3] X. Jin, R. Cheu, and D. Srinivasan, Development and adaptation of constructive probabilistic neural network in freeway incident detection, Transportation Research, 10C (2), 2002, pp. 121-147.

[4] D. Srinivasan, V. Sharma, and K. A. Toh, "Reduced multivariate polynomial-based neural network for automated traffic incident detection," Neural Netw., vol. 21, no. 2/3, pp. 484-492, Mar./Apr. 2008.

[5] F. Yuan and R. L. Cheu, "Incident detection using support vector machines," Transp. Res. C, Emerg. Technol., vol. 11, no. 3/4, pp. 309-328, Jun.-Aug. 2003.

[6] S. Chen,W.Wang, and H. VanZuylen, "Construct support vector machine ensemble to detect traffic incident," Expert Syst. Appl., vol. 36, no. 8, pp. 10 976-10 986, Oct. 2009.

[7] K. Zhang and M. A. P. Taylor, "Towards universal freeway incident detection algorithms," Transp. Res. C, Emerg. Technol., vol. 14, no. 2, pp. 68-80, Apr. 2006.

[8] K. Zhang and M. A. P. Taylor, "Effective arterial road inciden detection: A Bayesian network based algorithm," Transp. Res. C, Emerg. Technol., vol. 14, no. 6, pp. 403-417, Dec. 2006.

[9] S. M. Tang and H. J. Gao, "Traffic-incident detection-algorithm based on nonparametric regression," IEEE Trans. Intell. Transp. Syst., vol. 6, no. 1, pp. 38-42, Mar. 2005.

[10] H. Teng and Y. Qi, "Detection-delay-based freeway incident detection algorithms," Transp. Res. C, Emerg. Technol., vol. 11, no. 3/4, pp. 265-287, Jun.-Aug. 2003.

[11] W. Wei, C. Shuyan, and Q. Gaofeng, Comparison between partial least square and support vector machine for freeway incident detection, 10th international IEEE conference on intelligent transportation systems, Doubletree Hotel, Seattle, Washington, USA, 2007

[12] W. Wang, S. Chen, G. Qu, Incident detection algorithm based on partial least squares regression, Transportation Research Part C: Emerging Technologies, 16, 2008, pp. 54-70.

[13] H. J. Payne, E. D. Helfenbein, and H. C. Knobel, Development and testing of incident detection algorithms, Transportation Research Record, vol. 2, Report No. FHWA-RD-76-20, TRIS, 1976, pp. 316.

[14] J.F. Collins, C.M. Hopkins, and J.A. Martin, Automatic incident detection TRRL algorithms HIOCC and PATREG, TRRL Supplementary Report, No. 526, Crowthorne, Berkshire, U.K., 1979

[15] L. Lu, S. Chen, W. Wang, and Z. Zuylen, A hybrid model of partial least squares and neural network for traffic incident detection, Expert Systems with Applications, 2012, pp. 4775-4784,

[16] J. Wang, X. Li, S. Liao, and Z. Hua, A Hybrid Approach for Automatic Incident Detection, IEEE Transactions on Intelligent Transportation Systems, vol.14, 2013, pp. 1176-1185.

[17] E.Gakis, D.Kehagias, D.Tzovaras, "Mining traffic data for road incidents detection,", 2014 IEEE 17th International Conference on Intelligent Transportation Systems (ITSC), pp.930-935, 2014.

[18] K. F. Petty, "Incidents on the freeway: Detection and management," Ph.D. dissertation, Dept. Elect. Eng. Com. Sci., Univ. Calif. Berkeley, Berkeley, CA, USA, 1997.

[19] H. Al-Deek, Y. Fawaz, H. Noeimi, K. Petty, D. Rydzewski, K Sanwal, A. Skabardonis, and P. Varaiya. [Online]. Available: http://ipa.eecs.berkeley.edu/ pettyk/FSP/1995 